\title{
Enhancement of JPEG Compression for GPS Images
}

\author{
Yair Wiseman \\ Computer Science Department, Bar-Ilan University, Ramat-Gan 52900, Israel \\ wiseman@cs.biu.ac.il
}

\begin{abstract}
GPS devices typically make use of images that are excessively large to be stored as a Bit Map, so the images are always compressed. The widespread compression technique is JPEG; however JPEG has a disadvantage - JPEG assumes that the average color of beginning of each blocks line is commonly similar to the average color of end of its preceding blocks line. Almost always this assumption is wrong for GPS images. This paper proposes a method to adjust JPEG order of compression to an improved order that is more suitable for GPS images.
\end{abstract}

Keywords: Image Compression, JPEG, GPS

\section{Introduction}

The JPEG committee was established in 1986 by the CCITT and ISO Standards organizations with the aim of creating universal standards for image compression [1]. The committee finalized the standard by early 1991 and latter the standard was approved as an International Standards Organization (ISO). Initially, JPEG targeted achieving a 15:1 average compression ratio; however, currently JPEG achieves even better compression ratios. Some real-time, full-motion and video applications also used JPEG [2-3].

Many GPS applications use maps stored in small squares format [4]. With the aim of reducing transmitted data [5], these small squares are stored in the well known JPEG format [6]. The aim of this paper is presenting a better layout for the JPEG order of operation with the purpose of yielding a better compression result [7].

JPEG compression algorithm performs these operations:

At first, it transforms the image color into a suitable color space. The most common procedure is the converting into YUV components. These components are interleaved together within the compressed data. The ratio between these components is usually not one to one. The Y components will usually have a four times weight. The human eye is less sensitive to the frequency of chrominance information than to the frequency of luminance information which is represented by the Y component in the YUV format. Hence, the Y component gets a higher weight [8]. i.e. the order is $4 \mathrm{Y}$ blocks, one $\mathrm{U}$ block, one $\mathrm{V}$ block and then over and over again Y blocks, one U block, one $\mathrm{V}$ block; whereas each block contain $8 \mathrm{X} 8$ pixels.

JPEG employs Chroma subsampling which is a technique of encoding images by using less resolution for chrominance information than for luminance information, taking advantage of the human eye's lower sensitiveness for color differences than for luminance differences. JPEG supports the obvious 4:1:1 chroma subsampling which denotes the color resolution is quartered, compared to the luminance information i.e. for each sampled element as in figure 1, there is 4 numbers for luminance and just one number for chrominance [9]; however the default chroma subsampling of JPEG is 4:2:0 which denotes the horizontal sampling is doubled 
compared to $4: 1: 1$, but as the $\mathrm{U}$ and $\mathrm{V}$ components are only sampled on each alternate line.

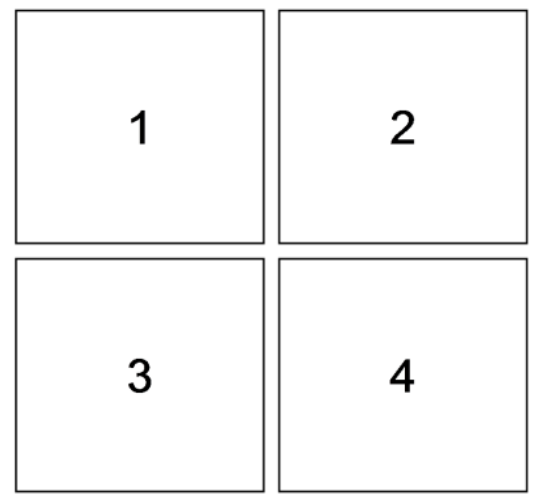

Figure 1. One JPEG Sampled Element

JPEG allows samples of 8 bits or 12 bits. In the original JPEG all values contained by the same source image must have the same precision. The values are shifted from unsigned integers with range $\left[0,2^{\mathrm{p}}-1\right]$ to signed integers with range $\left[-2^{\mathrm{p}-1}, 2^{\mathrm{p}-1}-1\right]$, by reducing $2 p-1$ from the original values, where $p$ can be either 8 or 12 [10]. These biased values are then sent to the next step.

The order of the blocks is line by line and each line is read from left to right. After the group into blocks, JPEG transforms each block through a Forward Discrete Cosine Transform (FDCT) [11]. The DCT gives a frequency map, with 8 X8 or 64 elements. The transformation keeps the low frequency information which a human eye is sensitive to [12].

Subsequently a quantization is done. In each block, each of the 64 coefficients is divided by a separate "quantization coefficient". The quantization coefficients are set according to the desired image quality. In point of fact, the image creator decides about a quality level (which is a number amongst 1 to 100) and according to this number the quantization coefficients are set. There is no standard method how to translate this quality level number into the quantization coefficients, so the quantization coefficients themselves are stored in the compressed image. The results of the division are rounded to integers. This step loses some information because of the rounding. Furthermore, it can be noted that even if the quantization coefficient is 1, some information will be lost, because more often than not the DCT coefficients are real numbers.

Finally, JPEG uses a Huffman compression method with the aim of reducing the size of the data. JPEG encodes the reduced coefficients using Huffman codes (Arithmetic coding [13-15] is also supported, but it is not commonly used). There are also other compression algorithms that fit JPEG, but are not officially supported [14]. 


\section{Improved JPEG based GPS Picture Compression}

As was mentioned above the blocks of JPEG are stored line by line. So, the first block of line no. $\mathrm{N}+1$ is stored after the last block of line no. N. Accordingly, the order of the block is as depicted in Figure 2:

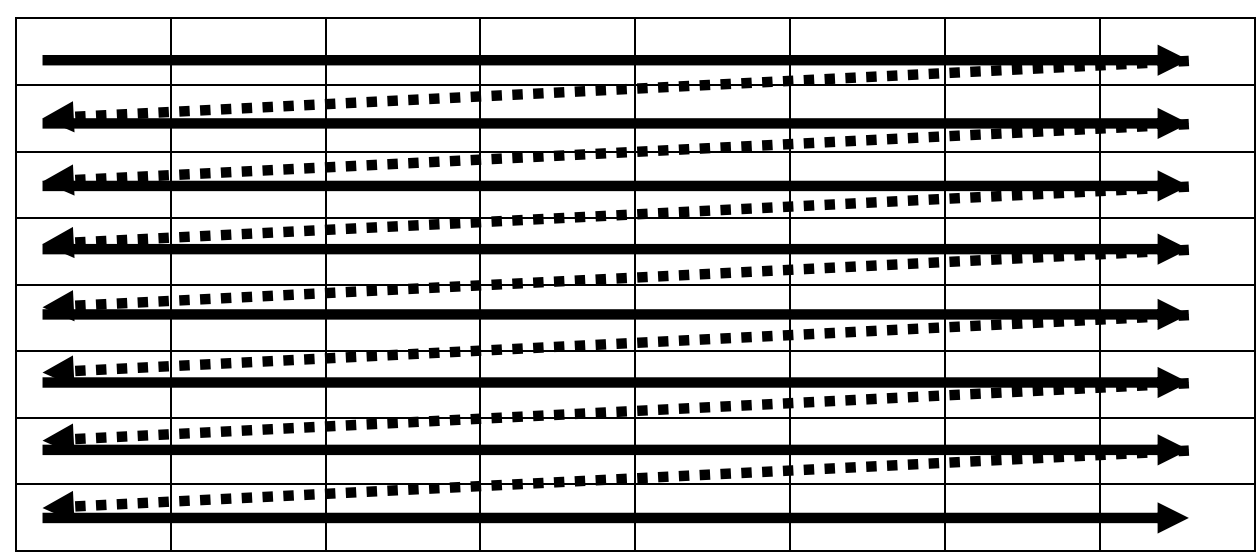

Figure. 2. The Order of the Lines in JPEG Image

The first value of each block is "DC" [15]. DC is the average intense of the block multiplied by 8 . It should be noted that DC is not stored as a plain value; rather it is stored as the difference between the current DC value and the previous DC value [16-17]. Usually, the DC values of adjacent blocks is similar [18-19], so it is commonsensical to store the difference which is a smaller number instead of the original number [20-22]; however the difference between the last DC in a line and the first DC in the next line is not always small because there are not DC values of adjacent blocks.

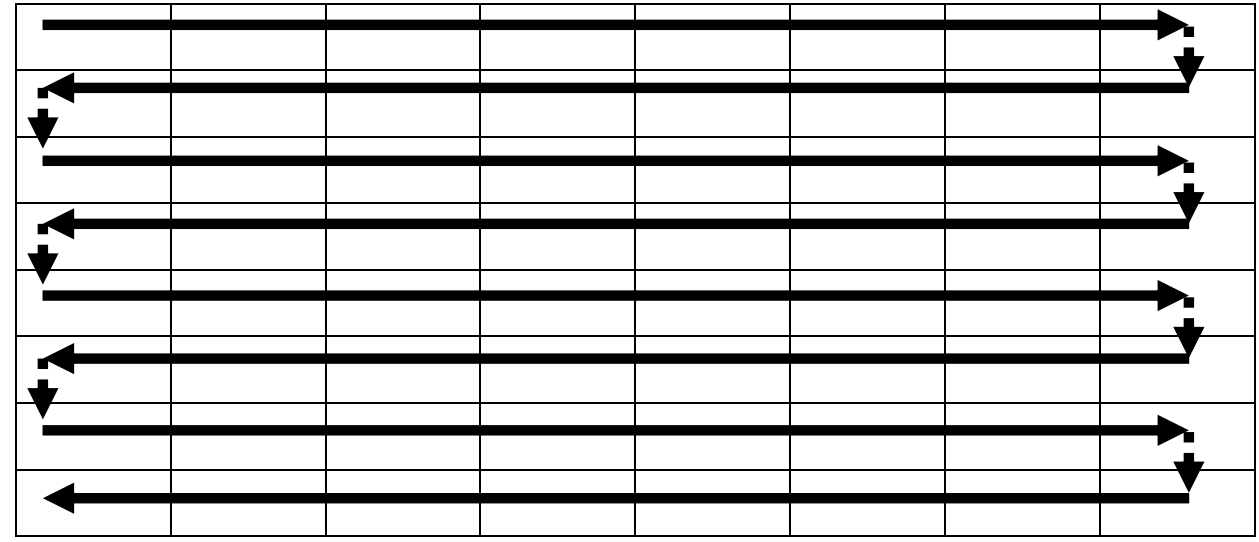

Figure. 3. New Order Line for JPEG Images.

The average colors of the beginning of the lines are typically quite similar and sometime are even equal. Likewise, the average colors of the end of the lines are also typically quite similar and sometime are even equal. The probability for the average colors of the beginning and the average colors of the end of the lines to be dissimilar is significantly higher [21-22]. For that reason, we suggest to change the order of the blocks in JPEG images to the order in Figure 3. In that way JPEG will always go through adjacent blocks and the probability of having similar DC values will become much higher; therefore the compression will be improved. 


\section{Experiments Results}

Figure 4 is a GPS map [23] of a region in Israel. Each line of the image's blocks is of 16 pixels height. Each block is $8 \mathrm{X} 8$ pixels, but since for each four Y block there are only one $\mathrm{U}$ block and one $\mathrm{V}$ block each four pixels of $\mathrm{U}$, just one pixel is actually stored and its value is the average of the four pixels [24]. The same is for V block. So for each 16X16 block of pixels there are four Y blocks, one $\mathrm{U}$ block and one $\mathrm{V}$ block.

In Figure 5 we can see the first and the second 16 pixels lines of the image. The average DC values of the last blocks of the first line and the average DC values of first block of the second line are shown in Table 1.

\begin{tabular}{|l|c|c|c|}
\hline & Last DC of First & $\begin{array}{c}\text { First DC of } \\
\text { Second line }\end{array}$ & Difference \\
\hline $\mathrm{Y}$ & 236 & 204 & -32 \\
\hline $\mathrm{U}$ & 122 & 156 & 34 \\
\hline $\mathrm{V}$ & 130 & 108 & -22 \\
\hline
\end{tabular}

Table 1. DC Values

As can be easily seen the average colors of the beginning of the lines is usually very similar and sometime is even identical. The likelihood for the average colors of the beginning and the end of the lines to be similar is to a great extent lower [25-26]. In particular, the differences between the average colors of the beginning of the lines in Figure 4 for $\mathrm{Y}, \mathrm{U}$ and $\mathrm{V}$ are all zero i.e. they are totally identical. The DC values of $\mathrm{Y}, \mathrm{U}, \mathrm{V}$ in the end of line 2 are specified in Table 2: 237, 125, 127, accordingly the differences between the average colors of the end of the lines in Figure 4 for $\mathrm{Y}$ is 1 , for $\mathrm{V}$ is 3 and for $\mathrm{V}$ is -3 .

In view of the fact that the DC values are stored as the differences between the current value and the previous value, we should focus on the differences. When there is no difference, according to [27], it will be indicated by JPEG by the sequence 00 which is a two bits string. A difference of 1 will be indicated by 0101 (4 bits), a difference of 3 will be indicated by 01111 ( 5 bits) and a difference of -3 will be indicated by 01100 ( 5 bits).

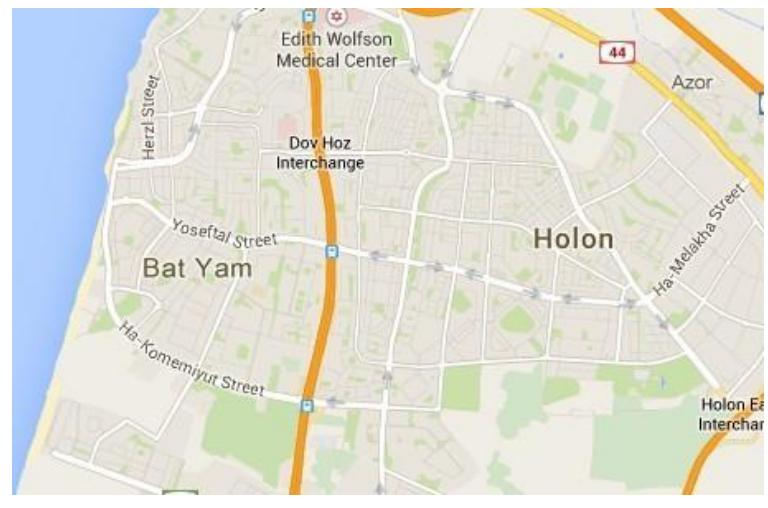

Figure. 4. Example of GPS Map

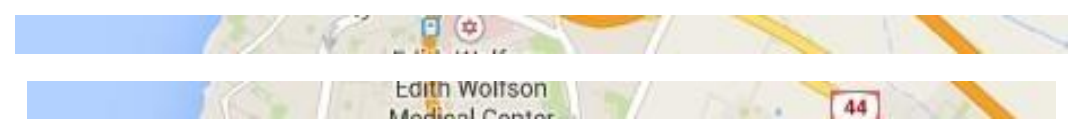

Figure. 5. Two First Lines of 16 Pixels 
On the other hand, in the original scheme of JPEG, -32 will be indicated by the string 1110011111 and 34 will be indicated by 1110100010 which are both 10 bits strings. -22 will be indicated by a slightly shorter bit string -11001001 which is just 8 bits, but still a longer string than the bit strings that represent the differences between the last DCs of the first line and the last DCs of the second line.

Table 2. DC Values.

\begin{tabular}{|c|c|c|c|}
\hline & $\begin{array}{c}\text { Last DC of } \\
\text { First line }\end{array}$ & $\begin{array}{c}\text { Last DC of } \\
\text { Second line }\end{array}$ & Difference \\
\hline $\mathrm{Y}$ & 236 & 237 & 1 \\
\hline $\mathrm{U}$ & 122 & 125 & 3 \\
\hline $\mathrm{V}$ & 130 & 127 & -3 \\
\hline
\end{tabular}

For that reason, we suggest to change the order of the blocks in JPEG images to the order in Figure 3. In the example of Figure 5 this will result in 14 bits $(4+5+5$ bits) instead of 28 bits $(10+10+8$ bits $)$ in the original scheme of JPEG. That is reduction of $50 \%$.

With the new suggested order the difference between the DCs of adjacent lines will be significantly smaller and the image will be compressed better. The changes between the DC values will be much more gradual; therefore the values of the differences will be much smaller resulting in smaller bit sequences for the codewords that the compressed data is consisting of.

The previous example in this section contained a sea; however, the differences in GPS images are not just because of water bodies. Yet another example is given herein below in Figure 6. This additional example contains green areas in the right side of the image and because of these areas, here again the differences between the DCs of the end of the first line and the DCs of the beginning of the second line are much higher than the differences between the DCs of the end of the first line and the DCs of the end of the second line.

Actually, this image has an interesting feature - The DCs of the end of the first line and the DCs of the end of the second line are exactly the same. In an arbitrary not adjacent blocks this can hardly ever happen; however, when the blocks are adjacent, the chance for such a case becomes quite good.

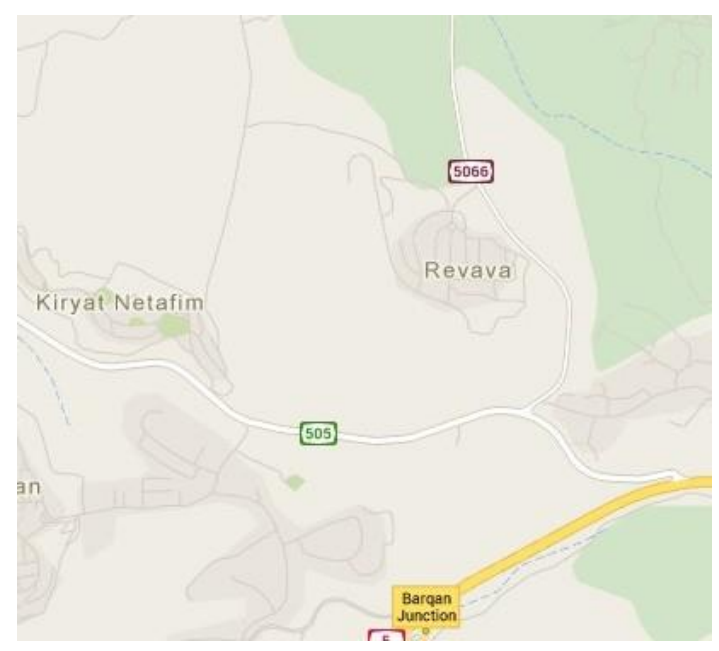

Figure 6. Yet Another Example of GPS Map 
Similarly to Figure 4, Figure 6 is a GPS map of a region in Israel whereas each line of the image's blocks is of 16 pixels height. The DC values of the end of the first line and DC values of the beginning of the second line and the differences between them are specified in Table 3 .

These values will be indicated by these code-words: 20 will be indicated by the string 11010100 ( 8 bits), 5 will be indicated by 100101 (6 bits) and 10 will be indicated by 1011010 (7 bits) which is in total 21 bits.

On the other hand, when there is no difference, it will be indicated by JPEG by the sequence 00 which is a two bits string; therefore, for 3 DCs the total needed bits is just 6 bits which is a reduction of $71.43 \%$.

Figure. 7. Two First Lines of 16 Pixels

Table 3. DC Values

\begin{tabular}{|l|c|c|l|}
\hline & $\begin{array}{c}\text { Last DC of } \\
\text { First line }\end{array}$ & $\begin{array}{c}\text { First DC of } \\
\text { Second line }\end{array}$ & Difference \\
\hline $\mathrm{Y}$ & 218 & 238 & 20 \\
\hline $\mathrm{U}$ & 117 & 122 & 5 \\
\hline $\mathrm{V}$ & 120 & 130 & 10 \\
\hline
\end{tabular}

Table 4. DC Values

\begin{tabular}{|l|c|c|l|}
\hline & $\begin{array}{c}\text { Last DC of } \\
\text { First line }\end{array}$ & $\begin{array}{c}\text { Last DC of } \\
\text { Second line }\end{array}$ & Difference \\
\hline $\mathrm{Y}$ & 218 & 218 & 0 \\
\hline $\mathrm{U}$ & 117 & 117 & 0 \\
\hline $\mathrm{V}$ & 120 & 120 & 0 \\
\hline
\end{tabular}

\section{Satellite Images}

Satellite images [28] usually have the same characteristic i.e. the possibility of the average colors of the beginning and the average colors end of the lines to be similar is extensively lower than the possibility of the average colors of adjacent blocks to be similar [29].

However, there is a noteworthy difference between satellite images and map images. Maps images typically make use of brighter colors whereas satellite images typically make use of both bright and dark colors [30]. This attribute to some extent reduces the possibility of the Y component differences to be large. 


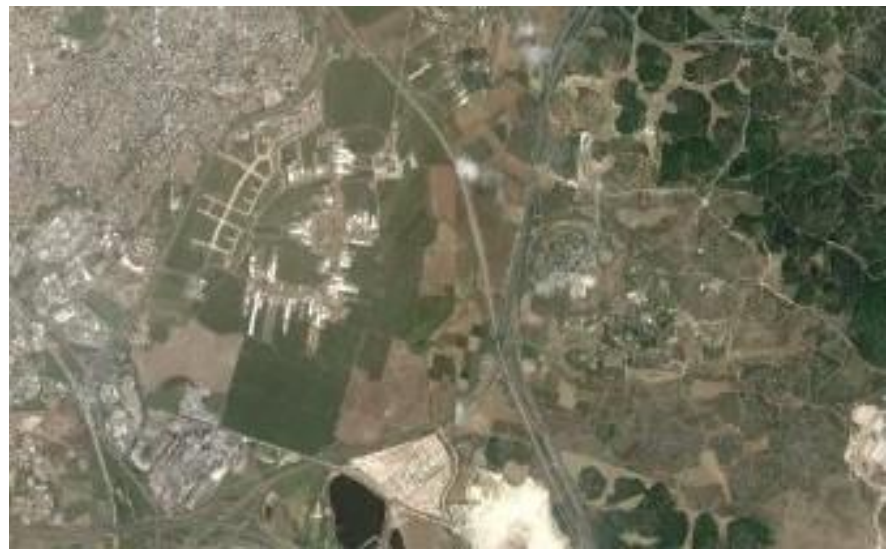

Figure. 8. Example of Satellite Image

Figure 8 is a good example for this attribute. The DC values of the end of the first line and DC values of the beginning of the second line and the differences between them are specified in Table 5. The difference between the DC values of the $\mathrm{Y}$ components is frequently very large as it is in this example. When the difference is calculated between the $\mathrm{Y}$ components of adjacent blocks, such a difference will hardly ever occur.

In this example the code-words for these values will be: 102 will be indicated by the string 111101100110 (12 bits), -4 will be indicated by 100011 (6 bits) and 10 will be indicated by 1011010 (7 bits) which is in total 25 bits.

When the order of the blocks is that the last block of the second line comes after the last block of the first line, the difference between the $\mathrm{Y}$ components is still relatively not so small, but it is not as large as the difference between the $\mathrm{Y}$ components in the original order.

The actual numbers for the new suggested order for this example are specified in Table 6 and they will be: 20 will be indicated by the string 11010100 ( 8 bits), 1 will be indicated by 0101 ( 4 bits) and -3 will be indicated by 01100 (5 bits) which is in total 17 bits and a reduction of $32 \%$.

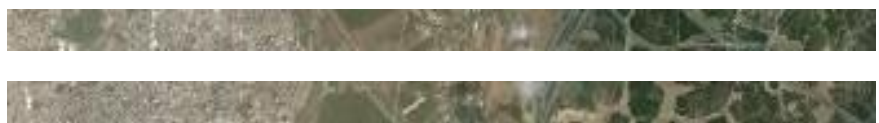

Figure. 9. Two First Lines of 16 Pixels

Table 5. DC Values

\begin{tabular}{|c|c|c|c|}
\hline & $\begin{array}{c}\text { Last DC of } \\
\text { First line }\end{array}$ & $\begin{array}{c}\text { First DC of } \\
\text { Second line }\end{array}$ & Difference \\
\hline $\mathrm{Y}$ & 62 & 167 & 102 \\
\hline $\mathrm{U}$ & 121 & 117 & -4 \\
\hline $\mathrm{V}$ & 125 & 135 & 10 \\
\hline
\end{tabular}

Table 6. DC Values

\begin{tabular}{|l|c|c|c|}
\hline & $\begin{array}{c}\text { Last DC of } \\
\text { First line }\end{array}$ & $\begin{array}{c}\text { Last DC of } \\
\text { Second line }\end{array}$ & Difference \\
\hline $\mathrm{Y}$ & 62 & 82 & 20 \\
\hline
\end{tabular}




\begin{tabular}{|l|l|l|l|}
\hline $\mathrm{U}$ & 121 & 122 & 1 \\
\hline $\mathrm{V}$ & 125 & 122 & -3 \\
\hline
\end{tabular}

\section{Conclusions}

GPS memory complicatedness [31-32] makes compression essential [33,34]. A technique of compression for JPEG based GPS picture with a higher compression ratio has been introduced. This paper has shown that JPEG's supposition that the average color of beginning of each blocks line is quite similar to the average color of end of its preceding blocks line is incorrect for most of GPS images. The change of the block order of JPEG compression algorithm can facilitate a reduction in the compressed file. The results are encouraging. The difference between the values of each blocks line can be dramatically decreased and as a result a much small compressed images can be obtained.

\section{References}

[1] Zhigang F. and De Queiroz R. L., "Identification of Bitmap Compression History: JPEG Detection and Qauntizer Estimation,” IEEE Transactions on Image Processing, vol. 12 no. 2, (2003), pp. 230-235.

[2] M. L. Main, D. Foltz, M. S. Firstenberg, E. Bobinsky, D. Bailey, B. Frantz, D. Pleva, M. Baldizzi, D. P. Meyers, K. Jones, M. C. Spence, K. Freeman, A. Morehead and J. D. Thomas, "Real-Time Transmission of Full-Motion Echocardiography over a High-Speed Data Network: Impact of Data Rate and Network Quality of Service," Journal of the American Society of Echocardiography, vol. 13 no. 8, (2000), pp. 764-770.

[3] P. H. Ang, P. A. Ruetz and D. Auld, "Video Compression Makes Big Gains," IEEE Spectrum, vol. 28 no. 10, (1991), pp. 16-19.

[4] L. Cao, J. Krumm, "From GPS traces to a routable road map," Proceedings of the 17th ACM SIGSPATIAL International Conference on Advances in Geographic Information Systems, Seattle, Washington, (2009), pp. 3-12.

[5] Y. Wiseman, K. Schwan and P. Widener, "Efficient End to End Data Exchange Using Configurable Compression," Proc. The 24th IEEE Conference on Distributed Computing Systems (ICDCS 2004), Tokyo, Japan, (2004), pp. 228-235.

[6] Sungjin H. and R. K. Hyune, "An Approach for the Similar File Detection with GPS Information," In First IEEE ACIS/JNU International Conference on Computers, Networks, Systems and Industrial Engineering (CNSI), Jeju Island, South Korea, (2011), pp. 320-324.

[7] Wiseman Y., "Improved JPEG Based GPS Picture Compression," Advanced Science and Technology Letters, (2015).

[8] S. H. Lee and I. C. Nam, "Intra prediction method based on the linear relationship between the channels for YUV 4:2:0 intra coding," 16th IEEE International Conference on Image Processing (ICIP- 2009), Cairo, Egypt, (2009), pp. 1037-1040.

[9] Levoy M., "Polygon-Assisted JPEG and MPEG Compression of Synthetic Images," In Proceedings of the 22nd ACM annual conference on Computer Graphics and Interactive Techniques, Los Angeles, CA, USA, (1995), pp. 21-28.

[10] Pennebaker W. B., “Adaptive quantization within the JPEG sequential mode,” U.S. Patent No. 5,157,488, issued October 20, (1992).

[11] Y. Wiseman, "The still image lossy compression standard - JPEG," Encyclopedia of Information and Science Technology, Third Edition, vol. 1, Chapter 28, (2014).

[12] Fleet D. J. and Heeger D. J., "Embedding Invisible Information in Color Images," In Proceedings of IEEE International Conference on Image Processing, Santa Barbara, CA, USA, vol. 1, (1997), pp. 532535.

[13] G. Feygin, P. G. Gulak and P. Chow, "Architectural Advances in the VLSI Implementation of Arithmetic Coding for Binary Image Compression," In Proceedings of IEEE Data Compression Conference (DCC'94), (1994), pp. 254-263.

[14] Y. Wiseman, "A Pipeline Chip for Quasi Arithmetic Coding," IEICE Journal - Trans. Fundamentals, Tokyo, Japan, vol. E84-A no.4, (2001), pp. 1034-1041.

[15] A. Moffat, M. N. Radford and Witten I. H., "Arithmetic Coding Revisited," ACM Transactions on Information Systems (TOIS), vol. 16 no. 3, (1998), pp. 256-294, pp.

[16] Y. Wiseman, "Burrows-Wheeler Based JPEG," Data Science Journal, vol. 6, (2007), pp. 19-27.

[17] M. I. Khan, J. Varun and M. A. Khan, "Perceptual Encryption of JPEG Compressed Images Using DCT Coefficients and Splitting of DC Coefficients into Bitplanes," In proceedings of IEEE International Conference on Intelligent and Advanced Systems (ICIAS), Kuala Lumpur, Malaysia, (2010), pp. 1-6. 
[18] S. T. Klein and Y. Wiseman, "Parallel Huffman Decoding," Proc. Data Compression Conference DCC2000, Snowbird, Utah, USA, (2000), pp. 383-392.

[19] S. T. Klein and Y. Wiseman, "Parallel Huffman Decoding with Applications to JPEG Files," The Computer Journal, Oxford University Press, Swindon, UK, vol. 46 no. 5, (2003), pp. 487-497.

[20] C. Y. Lin and Chang S. F., "A robust image authentication method distinguishing JPEG compression from malicious manipulation," IEEE Transactions on Circuits and Systems for Video Technology, vol. 11 no. 2, (2001), pp. 153-168.

[21] G. K. Wallace, "The JPEG still picture compression standard," IEEE Transactions on Consumer Electronics, vol. 38 no. 1, (1992), pp. 18-34.

[22] Y. Wiseman, "Take a Picture of Your Tire!" Proc. IEEE Conference on Vehicular Electronics and Safety (IEEE ICVES-2010) Qingdao, ShanDong, China, (2010), pp. 151-156.

[23] Y. Wiseman, "The Effectiveness of JPEG Images Produced By a Standard Digital Camera to Detect Damaged Tyres," World Review of Intermodal Transportation Research, vol. 4 no. 1, (2013), pp. 23-36.

[24] Y. Wiseman, "Camera That Takes Pictures of Aircraft and Ground Vehicle Tires Can Save Lives," Journal of Electronic Imaging, vol. 22 no. 4, (2013), pp. 041104.

[25] E. Fredj and Y. Wiseman, "An O(n) Algorithm for Edge Detection in Photos Compressed by JPEG Format," Proc. International Conference on Signal and Image Processing SIP-2001, Honolulu, Hawaii, (2001), pp. 304-308.

[26] Y. Wiseman and E. Fredj, "Contour Extraction of Compressed JPEG Images," ACM - Journal of Graphic Tools, vol. 6 no. 3, (2001), pp. 37-43.

[27] R. Gibson and E. Schuyler, "Google Maps Hacks," First Edition, O'Reilly Media Inc., Sebastopol, CA, USA, (2006).

[28] Maurer R. P., Barash D., Burgin R. A. and Thedens D. E., "Method Including Lossless Compression of Luminance Channel and Lossy Compression of Chrominance Channels," U.S. Patent no. 6,650,773, issued November 18, (2003).

[29] Y. Wiseman, "Fuselage Damage Locator System," Advanced Science and Technology Letters, vol. 37, (2013), pp. 1-4.

[30] Y. Wiseman, "Device for Detection of Fuselage Defective Parts," Information Journal, Tokyo, Japan, vol. 17 no. 9(A), (2014), pp. 4189-4194.

[31] B. W. Pennebaker and J. L. Mitchell, “JPEG: Still image data compression standard," Kluwer Academic Publisher, Norwell, MA, (2004).

[32] N. G. Kardoulas, A. C. Bird and A. I. Lawan, "Geometric Correction of SPOT and Landsat Imagery: A Comparison of Map and GPS Derived Control Points," Journal of Photogrammetric Engineering and Remote Sensing (PE\&RS), ASPRS, Bethesda, Maryland, USA, vol. 62 no. 10, (1996), pp. 1173-1177.

[33] R. Amato, G. Dardanelli, D. Emmolo, V. Franco, M. Lo Brutto, P. Midulla, P. Orlando and B. Villa, "Digital Orthophotos at a Scale of 1: 5000 from High Resolution Satellite Images," Journal of Photogrammetry \& Remote Sensing, Elsevier Publication for International Society for Photogrammetry and Remote Sensing (ISPRS), vol. 35, (2004), pp. 593-598.

[34] D. L. Verbyla, "Satellite Remote Sensing Of Natural Resources," CRC Press, Boca Raton, Florida, USA, (1995).

[35] D. Livshits and Y. Wiseman, "Cache Based Dynamic Memory Management for GPS," Proceedings of IEEE Conference on Industrial Electronics (IEEE ICIT-2011), Auburn, Alabama, (2011), pp. 441-446.

[36] D. Livshits and Y. Wiseman, "The Next Generation GPS Memory Management," International Journal of Vehicle Information and Communication Systems, vol. 3 no. 1, (2013), pp. 58-70.

[37] S. Worrall and E. Nebot, "Automated Process for Generating Digitized Maps Through GPS Data Compression.," In proceeding of Australasian Conference on Robotics and Automation, Brisbane, Australia, (2007).

[38] R. Steinmetz, "Data compression in multimedia computing - principles and techniques," Multimedia Systems, vol. 1 no. 4, (1994), pp. 166-172.

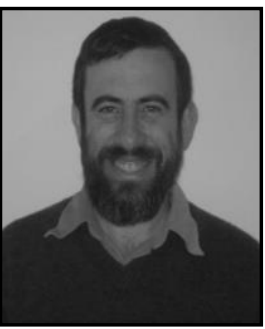

Yair Wiseman, he got a Summa Cum Laude M.Sc. and a $\mathrm{PhD}$ from Bar-Ilan University and completed two Post-Doc - one at the Hebrew University of Jerusalem and one in Georgia Institute of Technology. Dr. Wiseman's research interests include Computational Transportation Science, Intelligent Transportation Systems, Process Scheduling, Hardware-Software Codesign, Memory Management, Computer Clusters, Data Compression, JPEG, Embedded Systems, Real-Time Systems and Operating Systems. Dr. Wiseman is on the editorial board of several journals, a member of dozens of conference 
committees and a reviewer of many scholarly journals. Dr. Wiseman authored two books as well. In addition, Dr. Wiseman has been teaching in many institutes including Bar-Ilan University, The Hebrew University of Jerusalem, Israel Aircraft Industry, Holon Institute of Technology and Jerusalem College of Technology. Dr. Wiseman has been supervising many graduate students and an interesting point is that Albert Einstein is Dr. Wiseman's academic great-greatgrandfather (i.e. the advisor of the advisor of the advisor of Dr. Wiseman's advisor). Dr. Wiseman has collaborated with other partners and received research grants to run an active laboratory from inter alia Sun Microsystems, Intel, Polak Foundation and the Open University. Dr. Wiseman is an international expert who has reviewed and evaluated several large projects of the European Union, Israel Science Foundation, MB Logic and more. Dr. Wiseman's papers have been published in many venues around the world. 